\title{
On the Impact of Multi-mode Terminals in Heterogeneous Wireless Access Networks
}

\author{
X. Gelabert, J. Pérez-Romero, O. Sallent, R. Agustí \\ Departament de Teoria del Senyal i Comunicacions \\ Universitat Politècnica de Catalunya (UPC) \\ Barcelona, Spain \\ [xavier.gelabert, jorperez, sallent, ramon] @ tsc.upc.edu
}

\begin{abstract}
Beyond 3G (B3G) networks will encompass, among other features, a wide range of radio access technologies (RATs) providing users with a flexible and efficient access to the increasing pool of demanding services. This will allow users to get connected using the access technology that is most suitable according to some specified criteria. Consequently, to take full advantage of B3G networks, mobile terminals will need to support a larger set of capabilities. Among those, the support of different RATs, i.e. multi-mode capacity, is a must. This paper addresses the impact of multi-mode terminals in an EDGE/UMTS heterogeneous network with multi-service provisioning. Results indicate that multi-mode terminal availability should be considered when designing common radio resource management strategies in heterogeneous wireless access networks. Specifically, a service-based initial RAT selection policy is evaluated, revealing different behaviors for different multi-mode terminal availabilities and service-class mixings. In order to compensate the limitations imposed by non-multi-mode terminals, it is suggested to actuate over GERAN by using a resource reservation scheme for interactive users. By doing so, we tradeoff the QoS between multi-service/multi-mode users.
\end{abstract}

Keywords- Common radio resource management; multi-mode terminals; multi-access; heterogeneous networks; GERAN; UTRAN.

\section{INTRODUCTION}

Today's wireless communications comprise a broad variety of Radio Access Technology (RAT) standards. In Europe, the success of second-generation (2G) cellular system GSM (Global System for Mobile Communications) and the IP data connectivity support provided by GPRS (General Packet Radio System) paved the way towards evolved systems with higher data rate capabilities, such as the enhanced data rates for GSM evolution (EDGE) and finally the third-generation (3G) Universal Mobile Telecommunications System (UMTS) [1] [2]. Moreover, in parallel with the evolution of cellular systems, several types of Wireless Local Area Networks (WLANs) like, e.g., the IEEE 802.11x standard emerged and became profusely used in home environments. In order to provide the end-user with the requested service and corresponding QoS (Quality of Service) requirements in an Always Best Connected framework [3], beyond 3G (B3G) networks encompass the notion of integration and heterogeneity among different networks. In this way, heterogeneous networks may provide a larger set of available resources allowing users to seamlessly connect, at any time and

This work is partially funded by the IST-EVEREST project and by the COSMOS grant (ref. TEC2004-00518, Spanish Ministry of Science and Education and European Regional Development Fund). any place, to the access technology that is most suitable according to some user/operator specified criteria.

Under the previous statements, and in order to take full advantage of B3G networks, existing mobile terminal capabilities need to be extended. Particularly, to provide connectivity to a variety of underlying access technologies is a must. In this sense, multi-mode terminals, which are able to operate via different RATs, are devised [4]. The assumption that $2 \mathrm{G} / 2.5 \mathrm{G} / 3 \mathrm{G}$ multi-mode terminals are available for most users in 2009-2010 with a penetration reaching $90 \%$ is still valid [5]. Furthermore, it is expected that the penetration of multi-mode $2 \mathrm{G} / 2.5 \mathrm{G} / 3 \mathrm{G} / \mathrm{WLAN}$ terminals in the same timeframe will reach $50 \%$ of the population [5]. Therefore, in the short term, both single-mode ( $2 \mathrm{G}$ only) and multi-mode terminals will co-exist. On the other hand, the increasing complexity of multi-mode terminal devices may in turn result in a price increase. Consequently, some users may prefer simpler, smaller and cheaper devices for their basic needs such as, e.g., voice and short messaging service (SMS). Then, single-mode $2 \mathrm{G}$ or $2.5 \mathrm{G}$ terminals may not become extinct. All these facts, together with many other factors will cause differentiation in terminals and will cause segmentation in the terminal market to grow even further.

The Third Generation Partnership Project (3GPP) has identified several issues concerning multi-mode terminals. Specifically, [6] identifies multi-mode User Equipments (UE) categories as well as describes the general principles and procedures for the multi-mode operation. In [7] the parameters of the UE radio access capabilities are addressed and some reference configurations are provided for utilization in test specifications. As for considering multi-mode terminals in Radio Resource Management (RRM) procedures, load sharing among different RATs was already devised between $1 \mathrm{G}$ and $2 \mathrm{G}$ systems, like e.g., AMPS and CDMA-based IS-95, as a form of improving flexibility and lowering infrastructure costs. In [8] the expected gain obtained through statistical multiplexing effect (trunking gain) in multi-mode multicarrier CDMA/AMPS deployment is investigated by allocating multimode terminals to CDMA and single-mode terminals to AMPS. This study did not consider service differentiation when allocating users to different RATs, only voice calls and no vertical handovers (i.e. seamless roaming between RATs during call/session lifetime) either. More recently, Lincke et al. proposes in several papers, e.g. [9] and references therein, that capacity in a cellular network can be expanded by rearranging traffic (both voice and data) between different RATs, where 
only multi-mode terminals are capable of doing so. By means of reallocating multi-mode terminals (through vertical handover) new incoming users with single-mode terminal capabilities may experience lower blocking probabilities. Reference [9] compares several substitution policies and evaluates them by means of simulations.

This paper aims to analyse the impact of multi-mode terminal mixing in an EDGE/UMTS heterogeneous network with a policy-based initial RAT selection algorithm. This access selection is based on the demanding service-class and simulations will be performed considering different serviceclass mixings as well as multi-mode terminal mixings. The main contributions of this paper are: to show the impact of multi-mode terminal availability on the capacity of the system and to present an approach aiming to compensate the limitations exhibited by single-mode terminals via actuating over the configuration of GERAN by means of reserving resources for interactive users. Performance evaluation is carried out by means of extensive simulations in a highly detailed simulation scenario.

The remainder of the paper is organized as follows: Section II deals with the definition and capabilities of a multi-mode terminal in the context of a heterogeneous network with an initial RAT selection scheme. A detailed description of the simulation model and scenario is addressed in Section III. Simulation results and conclusions close the paper in Sections IV and $\mathrm{V}$ respectively.

\section{MulTi-MODE TERMINALS IN A COMMON RADIO RESOURCE MANAGEMENT FRAMEWORK}

According to [6], a multi-mode UE is considered to be a terminal with at least one UMTS Radio Access Mode (UTRA FDD and/or TDD). In addition, the multi-mode UE supports one or more other RATs, e.g., GSM, (E)GPRS, WLAN, etc. In particular, in our study we will consider multi-mode terminals to be those with connectivity to GERAN and UTRAN radio interfaces. On the contrary, single-mode terminals are those that support GERAN RAT only. Moreover, [6] defines several types of UEs, namely Type 1 through Type 4 . The assumed multi-mode terminal type in our paper is a Type 2 terminal, which can, when utilizing one RAT, perform monitoring of another RAT and report it using the current RAT.

To achieve a high utilisation of radio resources in a heterogeneous access network, Common RRM (CRRM) strategies are defined to manage them in an optimum way [10]. Along with other tasks, a CRRM entity is devised to carry out the RAT selection procedure either at the beginning of the session or during the session lifetime, i.e. in the case of a vertical handover. A solution where initial RAT selection is performed according to certain policies is adopted in [11]. Based on a given set of input parameters, policies can make decisions in order to allocate users to a particular RAT. In this paper, we will assume that such decisions are based on the demanding service-class where a mix of voice and interactive users is considered. Specifically, this service policy first attempts to assign voice users to GERAN and interactive users to UTRAN. If no capacity is available in GERAN, voice users try admission to UTRAN. Similarly, rejected interactive users in UTRAN will attempt admission in GERAN. If no capacity is available in any of the RATs, the user gets blocked. Note that all this will apply provided the terminal has the required capabilities to operate with the suitable RAT, otherwise GERAN is selected as the default RAT. Let Fig. 1 illustrate the initial RAT selection policy considering multi-mode terminal capabilities.

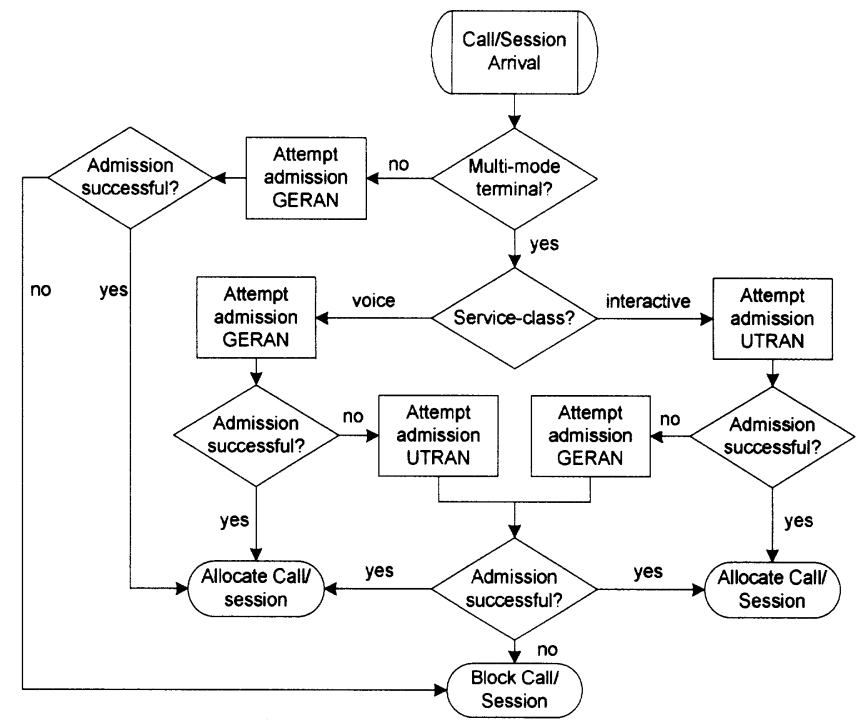

Figure 1. Initial RAT selection flow chart considering multi-mode terminals.

\section{SiMULATION ENVIRONMENT}

A scenario with UTRAN and GERAN access technologies is considered. We assume a $2.25 \times 2.25 \mathrm{~km}^{2}$ area with 7 collocated omnidirectional cells for GERAN and UTRAN. Sites are separated a distance of $1 \mathrm{~km}$. It is assumed for GERAN that the 7 cells represent a cluster where each cell works with different carrier frequencies. Three carriers per cell, belonging to the $1800 \mathrm{MHz}$ band, are used in GERAN, while a single carrier is considered in UTRAN. The urban macrocell model is assumed with shadowing deviation of $10 \mathrm{~dB}$ [12]. Table I shows the some of the considered simulation parameters.

A service-class mix of voice and interactive (data) users is considered moving at $3 \mathrm{~km} / \mathrm{h}$. Voice calls are generated according to a Poisson process with an average call rate of 10 calls/h/user and exponentially distributed call duration with an average $180 \mathrm{~s}$. In UTRAN, the RAB for voice users is the 12.2 $\mathrm{kb} / \mathrm{s}$ speech one defined in [13], considering a dedicated channel $(\mathrm{DCH})$ with spreading factor 64 in the uplink and 128 in the downlink. As for GERAN, voice users are allocated to a TCH-FS (traffic channel with full-rate speech), i.e. one time slot in each GSM frame.

Interactive users follow the www browsing model given in [14], with 5 pages per session, an average reading time between pages of $30 \mathrm{~s}$, an average of 25 objects (packets) per page, and inter-arrival packet time $0.125 \mathrm{~s}$ for the UL and $0.0228 \mathrm{~s}$ for the DL. The average packet size is 366 bytes. This leads to an average bit rate during activity periods of $24 \mathrm{~kb} / \mathrm{s}$ in the uplink and $128 \mathrm{~kb} / \mathrm{s}$ in the downlink. A session rate of 24 sessions/h/user is assumed. The web browsing service is provided in UTRAN by means of DCH making use of 


\begin{tabular}{|c|c|c|c|}
\hline \multicolumn{2}{|c|}{ UTRAN BS parameters } & \multicolumn{2}{|c|}{ UTRAN RRM parameters } \\
\hline Max. transmitted power & $43 \mathrm{dBm}$ & Admission method UL & Based on uplink load factor \\
\hline Thermal noise & $-104 \mathrm{dBm}$ & Admission method DL & Based on transmitted power \\
\hline Common Control Channels Power & $33 \mathrm{dBm}$ & UL admission threshold $\left(\eta_{\max }\right)$ & 1.0 \\
\hline Max. DL power per user & $41 \mathrm{dBm}$ & DL admission threshold $\left(\mathrm{P}_{\max }\right)$ & $42 \mathrm{dBm}$ \\
\hline \multicolumn{2}{|c|}{ UTRAN UE parameters } & Active Set size & 1 \\
\hline Max. transmitted power & $21 \mathrm{dBm}$ & Replacement hysteresis & $3 \mathrm{~dB}$ \\
\hline Min. transmitted power & $-44 \mathrm{dBm}$ & Time to trigger handover & $0.64 \mathrm{~s}$ \\
\hline Thermal noise & $-100 \mathrm{dBm}$ & \multicolumn{2}{|c|}{ GERAN RRM parameters } \\
\hline DL Orthogonality factor & 0.4 & Link adaptation period & $1 \mathrm{~s}$. \\
\hline \multicolumn{2}{|c|}{ GERAN BS parameters } & BS_CV_MAX & 15 \\
\hline DL transmitted power & $43 \mathrm{dBm}$ & GPRS_MS_TXPWR_MAX_CCH & $43 \mathrm{dBm}$ \\
\hline Thermal noise & $-117 \mathrm{dBm}$ & GPRS RESELECT OFFSET & $-2 \mathrm{~dB}$ \\
\hline Number of carriers & 3 & GPRS_RXLEV_ACCESS MIN & $-105 \mathrm{dBm}$ \\
\hline EGPRS slots & All reversible except slot 0 of first carrier & Max. number of TBFs per slot & UL: 8, DL:32 \\
\hline \multicolumn{2}{|c|}{ GERAN UE parameters } & L RXLEV UL_H & $-100 \mathrm{dBm}$ \\
\hline Max. transmitted power & $33 \mathrm{dBm}$ & L_RXLEV DL $\mathrm{H}$ & $-100 \mathrm{dBm}$ \\
\hline Min. transmitted power & $0 \mathrm{dBm}$ & MS_RANGE_MAX & $35 \mathrm{~km}$ \\
\hline Thermal noise & $-113 \mathrm{dBm}$ & P5 & 3 \\
\hline Multislot class & $2 \mathrm{UL}, 3 \mathrm{DL}, 4 \mathrm{UL}+\mathrm{DL}$ & P8 & 3 \\
\hline
\end{tabular}

transport channel type switching to RACH/FACH during inactivity periods. The considered RAB assumes a maximum bit rate in the uplink of $64 \mathrm{~kb} / \mathrm{s}$ (corresponding to a minimum spreading factor of 16) and in the downlink of $128 \mathrm{~kb} / \mathrm{s}$ (with a spreading factor of 16). The RAB characteristics are given in [13]. On the other hand, the www service in GERAN is provided through a PDCH (Packet Data Channel) shared among users through round robin scheduling. A link adaptation mechanism selects the highest modulation and coding scheme (MCS) that ensures the CIR requirements. The considered maximum allowed MCS in our study is MCS-7, corresponding to a bit rate of $44.8 \mathrm{~kb} / \mathrm{s}$ per time slot. Then, assuming that the multislot class allows up to 2 uplink slots and 3 downlink slots (see Table I), the maximum bit rate is $89.6 \mathrm{~kb} / \mathrm{s}$ in the uplink and $134.4 \mathrm{~kb} / \mathrm{s}$ in the downlink. Consequently, in terms of maximum bit rate, similar values are considered for both UTRAN and GERAN, thus enabling consistent comparisons.

Considered QoS parameters set the BLER target at $1 \%$ and $10 \%$ for voice and interactive users respectively in both RATs. Dropping occurs in UTRAN when BLER is $1 \mathrm{~dB}$ below target during $20 \mathrm{~s}$. In GERAN, dropping happens when BLER is $5 \mathrm{~dB}$ below target during $20 \mathrm{~s}$. or when 10 consecutive unsuccessful $\mathrm{HO}$ retries take place.

\section{Simulation Results}

In the following, the performance of the previously defined initial RAT selection policy considering different multi-mode terminal availabilities and service mixing is evaluated by means of simulation. In particular, representative values of multi-mode terminal availabilities consider, $25 \%, 50 \%, 75 \%$ and $100 \%$, which indicate the percentage of terminals that support both RATs, i.e. GERAN and UTRAN. As for the service class mixing, several sets consisting of voice and interactive users are considered. Let $\vec{u}_{i}=(V U, W U)$ represent a service-class mixing set $i$ consisting of a number of voice users (VU) and interactive users (WU). It is worth noticing that strain is placed on GERAN, not only having to cope with voice users being assigned by the aforementioned policy, but also with interactive users having single-mode (only GERAN) terminals.

\section{A. Throughput Perfromance.}

Table II shows the total aggregated throughput for different values of multi-mode terminal availability and different sets of service class mixings. A first expected result is that maximum throughput is achieved, for each service mix set, when all terminals are multi-mode (see rightmost column in Table II). Results show that, as long as GERAN can handle its share of users, i.e. voice and single-mode terminal interactive users, no throughput degradation is noted when decreasing the number of multi-mode terminals. This is the case, e.g. for $V U=200$.

TABLE II. TOTAL UL AGgREGATE ThroughPUT (MB/s).

\begin{tabular}{c|c|c|c|c|c}
\hline \multicolumn{3}{c|}{$\overrightarrow{\mathbf{u}}_{\mathbf{i}}=\left(\mathbf{V U}_{\mathbf{j}}, \mathbf{W U}_{\mathbf{j}}\right)$} & \multicolumn{4}{c}{ Multi-mode Terminal Availability (\%) } \\
\hline $\mathbf{V U}_{\mathbf{j}}$ & $\mathbf{W U}_{\mathbf{j}}$ & $\mathbf{2 5}$ & $\mathbf{5 0}$ & $\mathbf{7 5}$ & $\mathbf{1 0 0}$ \\
\hline \multirow{4}{*}{$\mathbf{2 0 0}$} & $\mathbf{2 0 0}$ & 1,35 & 1,37 & 1,39 & 1,39 \\
\cline { 2 - 6 } & $\mathbf{4 0 0}$ & 1,76 & 1,79 & 1,77 & 1,81 \\
\cline { 2 - 6 } & $\mathbf{6 0 0}$ & 2,18 & 2,18 & 2,19 & 2,20 \\
\hline \multirow{4}{*}{$\mathbf{4 0 0}$} & $\mathbf{2 0 0}$ & 2,06 & 2,10 & 2,14 & 2,17 \\
\cline { 2 - 6 } & $\mathbf{4 0 0}$ & 2,29 & 2,40 & 2,50 & 2,55 \\
\cline { 2 - 6 } & $\mathbf{6 0 0}$ & 2,41 & 2,71 & 2,87 & 2,97 \\
\hline \multirow{4}{*}{$\mathbf{6 0 0}$} & $\mathbf{2 0 0}$ & 2,17 & 2,39 & 2,55 & 2,70 \\
\cline { 2 - 6 } & $\mathbf{4 0 0}$ & 2,27 & 2,57 & 2,87 & 3,07 \\
\cline { 2 - 6 } & $\mathbf{6 0 0}$ & 2,38 & 2,78 & 3,19 & 3,46 \\
\hline
\end{tabular}

Since the maximum aggregated throughput is achieved in each service class mixing for $100 \%$ of multi-mode terminal availability, it can be useful to measure the degradation introduced by single-mode terminals. Accordingly, we define the throughput degradation $D_{i, j}$ for a given service class mixing $i$ and a multi-mode terminal availability $j$ as:

$$
D_{i, j}(\%)=\frac{C_{100}^{i}-C_{j}^{i}}{C_{100}^{i}} \cdot 100
$$


where $C_{j}^{i}$ is the total aggregated throughput for service class mixing $i$ and multi-mode terminal availability $j$.

Fig. 2 shows the throughput degradation as defined previously. Notice that, for different multi-mode terminal availabilities and different number of users requesting service, throughput degradation exhibits different trends. In particular, for $\vec{u}=(200,200)$, no big differences are noticed, meaning that, in this case, GERAN is able to manage voice and singlemode terminals with ease. Certainly, the average timeslot utilisation factor in GERAN (properly defined in [1]) reveals an occupation of resources below $70 \%$. The increase of users requesting to be served is translated into a bigger degradation in terms of throughput as multi-mode terminal availability decreases. While for $\vec{u}=(400,400)$ degradation starts to get noticeable for multi-mode availabilities of $25 \%$ and $50 \%$, for $\vec{u}=(600,600)$ this degradation is already perceptible at $75 \%$ of multi-mode availability.

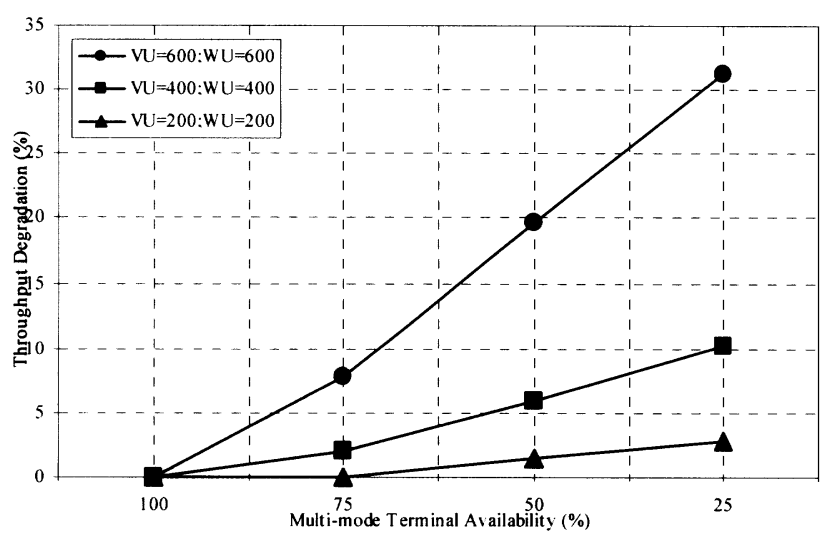

Figure 2. Uplink throughput degradation due to multi-mode terminals.

\section{B. Delay Performance}

Packet delay statistics for interactive users also reveal degradation when considering a scenario with mixed multimode and single-mode terminals. This degradation impacts directly in the perceived QoS by the data user and it is therefore important to keep its value as low as possible.

Fig. 3 shows the uplink average packet delay for interactive users being served through GERAN for different mixings of multi-mode terminals and service-classes. Notice the increasing packet delay when multi-mode terminal availability decreases for $\vec{u}=(400,400)$. As for $\vec{u}=(200,200)$, the average packet delay remains almost constant with multi-mode terminal availability and also at an acceptable level, therefore exhibiting no degradation in this sense. Recall that the same behavior was also observed when analyzing throughput performance earlier on. A look at the average timeslot utilization factor reveals that, for $\vec{u}=(400,400)$, this value is over $90 \%$ while for $\vec{u}=(200,200)$ this value is kept below $70 \%$. This explains the big difference between the average packet delays of both service mixings. Observe that a multimode terminal availability of $100 \%$ is not taken into account because the considered service policy does not allocate any, or hardly any, interactive user in GERAN, so no statistics are available in this case.

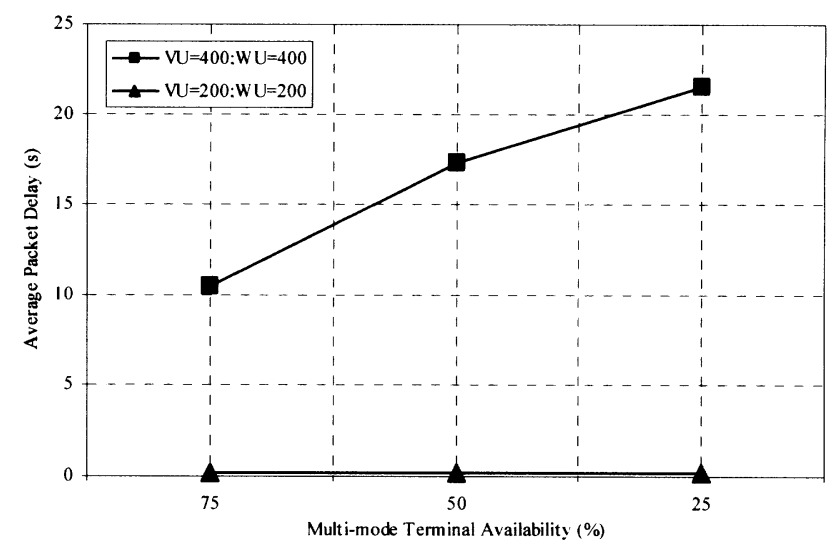

Figure 3. Uplink average packet delay for interactive users.

\section{Approach using EGPRS dedicated slots}

The suggested approach to overcome the delay increase problem, shown in Fig. 3, resides in the introduction of dedicated EGPRS timeslots for interactive users. In this way, some resources may be reserved for interactive users and therefore packet delay performance can be improved with respect to not having any reservation scheme. As for throughput performance, it can be foreseen that contribution of voice users to the total aggregate throughput might diminish. This reduction of voice throughput may be compensated, given certain conditions, by interactive users allocated to the reserved slots. This entails a trade-off between the number and the applicability of reserved resources (slots) for interactive users in GERAN and other parameters, such as offered load and multi-mode terminal availability, as we will see in the following.

Without loss of generality we assume a resource reservation scheme by dedicating 3 EGPRS slots per cell for interactive users. Recall that, three carriers per cell are available and that slot 0 of first carrier is devoted to control and signaling. Thus, 23 slots are left of which 3 are EGPRS-only slots (13\% of total resources) and the rest are reversible slots for both voice and interactive services ( $87 \%$ of total resources).

In order to evaluate the suitability of using dedicated slots, we can define a gain parameter, $G$, as:

$$
G_{i, j}(\%)=\frac{C_{0}^{i . j}-C_{3}^{i . j}}{C_{3}^{i . j}} \cdot 100
$$

with $C_{k}^{i . j}$ being the total aggregated throughput for service mixing set $i$, multi-mode availability $j$ and $k$ dedicated slots for EGPRS services. This gain indicates if aggregate throughput performance is improved $(G>0)$, degraded $(G<0)$ or unconcerned ( $G=0$ ), when using 3 dedicated EGPRS slots for data traffic as opposed to not using any.

Table III shows the gain introduced by dedicated resources as defined in (2). It can be seen that for 200 voice users and the considered set of interactive users no especial improvement or 
degradation is noted. This is because GERAN can manage with 200 voice users even with the shortage of resources introduced by dedicated data slots and still can also handle single-mode terminal users requesting interactive service either by allocating them to dedicated slots or to reversible slots. On the other hand, UTRAN is able to serve the set of multi-mode terminal interactive users and therefore does not make use of resources in GERAN. Thus, no degradation or improvement is observed in terms of throughput.

As for 400 voice users, the improvement in throughput due to the reservation scheme depends on both the service-class mixing and the multi-mode terminal availability. In general, the overall trend when the number of multi-mode terminals increases is reflected in the gain reduction of throughput due to the reservation scheme. In particular, for $\vec{u}=(400,200)$, degradation in throughput is observed, which gets more severe as the number of multi-mode terminals increases. On the contrary, increasing the number of interactive users results in a throughput gain, particularly for low multi-mode terminal availability. This is explained due to the fact that, for voice loads above $87 \%$ (i.e. the percentage of reversible resources), gain is only achieved if the interactive users are sufficiently high in order to contribute with throughput in the remaining $13 \%$ of dedicated resources and compensate for the lack of voice throughput contributions in those reserved resources.

For 600 voice users, the behaviour is similar to the case of 400. However, resource reservation gain is achieved even for higher multi-mode terminal availabilities.

TABLE III. EGPRS SLOT RESERVATION GAIN (\%).

\begin{tabular}{c|c|c|c|c|c}
\hline \multicolumn{2}{c|}{$\overrightarrow{\mathbf{u}}_{\mathbf{i}}=\left(\mathbf{V U}_{\mathbf{j}}, \mathbf{W U}_{\mathbf{j}}\right)$} & \multicolumn{4}{c}{ Multi-mode Terminal Availability (\%) } \\
\hline $\mathbf{V U}_{\mathbf{j}}$ & $\mathbf{W U}_{\mathbf{j}}$ & $\mathbf{2 5}$ & $\mathbf{5 0}$ & $\mathbf{7 5}$ & $\mathbf{1 0 0}$ \\
\hline \multirow{4}{*}{$\mathbf{2 0 0}$} & $\mathbf{2 0 0}$ & 0.00 & 0.00 & 0.72 & 0.00 \\
\cline { 2 - 6 } & $\mathbf{4 0 0}$ & 1.14 & 0.56 & 1.13 & 0.55 \\
\cline { 2 - 6 } & $\mathbf{6 0 0}$ & 0.00 & 0.46 & -0.46 & -0.91 \\
\hline \multirow{4}{*}{$\mathbf{4 0 0}$} & $\mathbf{2 0 0}$ & -3.88 & -4.76 & -6.07 & -7.83 \\
\cline { 2 - 6 } & $\mathbf{4 0 0}$ & 3.49 & -0.42 & -4.40 & -5.10 \\
\cline { 2 - 6 } & $\mathbf{6 0 0}$ & 8.71 & 2.21 & -1.39 & -4.71 \\
\hline \multirow{4}{*}{$\mathbf{6 0 0}$} & $\mathbf{2 0 0}$ & 1.38 & -2.10 & -2.75 & -1.48 \\
\cline { 2 - 6 } & $\mathbf{4 0 0}$ & 13.22 & 7.00 & -0.35 & -4.23 \\
\cline { 2 - 6 } & $\mathbf{6 0 0}$ & 13.87 & 12.23 & 3.45 & -1.16 \\
\hline
\end{tabular}

Finally, Fig. 4 depicts the uplink average packet delay when considering 3 dedicated EGPRS slots for interactive users. Clearly, these users benefit from the dedicated slots exhibiting lower packet delays than in the case of not having any reservation scheme (see Fig. 3).

\section{CONCLUSIONS}

This paper has covered several aspects on the impact of multi-mode terminals in the framework of heterogeneous networks with an initial RAT selection policy. Results indicate degradation in terms of throughput introduced by the limited operation of single-mode terminals. By considering a reservation scheme in GERAN for interactive users, we can improve the average packet delay for such users. While for a high multi-mode terminal availability results indicated that the reservation scheme was not necessary, for lower multi-mode terminal availabilities this scheme improved both aggregated throughput and packet delay figures, particularly for high number of interactive users.

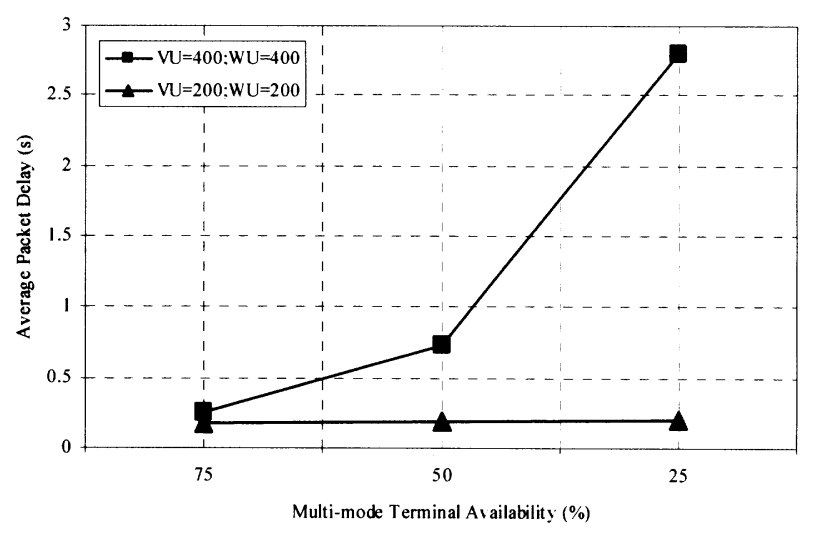

Figure 4. Uplink average packet delay for interactive users for different multi-mode terminal availability and 3 dedicated EGPRS slots.

\section{REFERENCES}

[1] T. Halonen, J. Romero, and J. Melero (editors), GSM, GPRS and EDGE performance : evolution towards 3G/UMTS, Wiley, 2002.

[2] H. Holma and A.Toskala (editors), WCDMA for UMTS Radio Access For Third Generation Mobile Communications, Wiley, 2001.

[3] E. Gustafsson and A. Jonsson, "Always best connected," IEEE Wireless Communications, vol. 10, no. 1, pp. 49-55, Feb. 2003.

[4] R.E. Schuh, P. Eneroth, and P. Karlsson, "Multi-Standard Mobile Terminals," Proceedings of the IST Mobile \& Wireless Telecommunications Summit '02, pp. 174-178, June 2002.

[5] P. Karlsson (editor) et al. "Target Scenarios specification: vision at project stage 2 Deliverable D13 of the EVEREST IST-2002-001858 project, February, 2005. Available: http://www.everest-ist.upc.es

[6] 3GPP TR 21.910 3.0.0, "Multi-mode UE issues; categories, principles and procedures".

[7] 3GPP TR 25.306 5.9.0, "UE Radio Access capabilities definition”.

[8] T.W. Wong and V.K. Prabhu, "Multi-Mode / Multi-Carrier Resource Management in CDMA / AMPS Deployment", IEEE 49th Vehicular Technology Conference, 1999, Vol. 3, pp. 1861-1865.

[9] S. J. Lincke "Vertical handover policies for common radio resource management", International Journal of Communication Systems 2005; Published Online: 15 Mar 2005. DOI: 10.1002/dac.715.

[10] J. Pérez-Romero, O. Sallent, R. Agustí and M.A. Díaz-Guerra, Radio Resource Management strategies in UMTS, Wiley, 2005.

[11] J. Pérez-Romero, O. Sallent and R. Agustí, "Policy-based Initial RAT Selection algorithms in Heterogeneous Networks" accepted at MWCN 05. Marrakech-Morocco 19-21 Sept. 2005

[12] 3GPP TR 25.942, v5.1.0 "RF System Scenarios, Release 5. (2002 06)".

[13] 3GPP TS 34.108 "Common Test Environments for User Equipment (UE); conformance testing".

[14] UMTS 30.03 v3.2.0 TR 101112 "Selection procedures for the choice of radio transmission technologies of the UMTS", ETSI, April, 1998. 